\title{
Assessment of single-turn extraction for a superconducting separated-sector cyclotron
}

\author{
J.-W. Kim, A. Goto, T. Mitsumoto, and Y. Yano \\ Cyclotron Laboratory, Institute of Physical and Chemical Research (RIKEN), Wako, Saitama 351-0198, Japan
}

(Received 17 July 1998; published 22 April 1999)

\begin{abstract}
We have studied the beam dynamics in a high field separated-sector cyclotron to establish conditions required to achieve single-turn extraction. Extraction efficiency above $99 \%$ is aimed to avoid beaminduced damages. A sufficient last-turn separation and a preservation of beam quality throughout the acceleration are the prerequisites. The last-turn separation can be enhanced with the orbital precession induced by an off-centering injection as in existent lower field separated-sector cyclotrons. The beam qualities, on the other hand, are affected by the resonances traversed or approached during the acceleration. Acceleration of light nuclei to the energies of 300-400 MeV/u was especially disturbing because of the broadening of radial beamwidth upon crossing the $\nu_{r}=3 / 2$ resonance. Tolerance to the third harmonic gradient field, which causes the resonance in lowest order, is set to be $0.1 \mathrm{G} / \mathrm{cm}$. The integer resonance $\nu_{z}=1$ can be avoided with a proper design of the yoke. The longitudinal space charge forces were also investigated to estimate the intensity limits determined by the energy spreads of beam assuming simplified charge distributions. Moreover, beam preparations for injection matching are described in view of reducing the beam losses into the extraction elements. [S1098-4402(99)00036-1]
\end{abstract}

PACS numbers: $29.20 . \mathrm{Hm}$

\section{INTRODUCTION}

A superconducting ring cyclotron (SRC) which is under construction at RIKEN will accelerate high-current heavy ion beams to produce radioisotope (RI) beams through a projectile fragmentation process [1]. The $K$ value of the cyclotron was chosen to be $2500(q / A)^{2} \mathrm{MeV} / \mathrm{u}$ in order to achieve the maximum design energy of $400 \mathrm{MeV} / \mathrm{u}$ for light nuclei with the charge to mass ratio around 0.5 and of $150 \mathrm{MeV} / \mathrm{u}$ for heavy nuclei with that ratio nearly 0.25 . The average injection radius is $353 \mathrm{~cm}$, and extraction radius $536 \mathrm{~cm}$. The ratio of the two radii corresponds to the velocity gain. The main components of the cyclotron are six units of sector magnets, four singlegap cavities, and a third harmonic flat-topping cavity. The superconducting sector magnet which is a core part of this cyclotron has been prototyped, and will be tested in the near future [2].

The main cavity will be operated by the radio frequencies variable in the range from 18 to $38 \mathrm{MHz}$ in the sixth harmonic of the particle revolution frequency. The shunt impedance of the cavity becomes higher at higher frequencies, which enables a larger energy gain per turn for the light nuclei. The total number of turns is larger for the light nuclei being accelerated from 126 to $400 \mathrm{MeV} / \mathrm{u}$ because the total energy gain of the light nuclei is larger than that of the heavy nuclei. Those light nuclei are thus the main design particles in the beam extraction study.

The turn separation at extraction should be larger than the beamwidth for the purpose of single turn extraction. Energy gain alone cannot provide a sufficient turn separation in usual cyclotrons. Various methods may be applicable to amplify the last-turn separation depending on the operation regime of each cyclotron [3]. For the SRC in which $\nu_{r}$ is remote from unity because of high injection energies, the most effective method is an off-centering injection. A precession which is induced at the injection due to orbital off-centerednesss proceeds until the extraction without deteriorating the beam quality.

Resonance crossing during the acceleration may degrade the beam quality. Orbit tracking has been performed to study the orbit behaviors of design particles using computed magnetic fields. The isochronous magnetic fields were produced by fitting the main and trim coil currents using the field form factors calculated at several different excitations [4] with TOSCA [5]. The focusing tunes of design particles are depicted in Fig. 1, where the plots are smoothed to reduce the irregularities caused

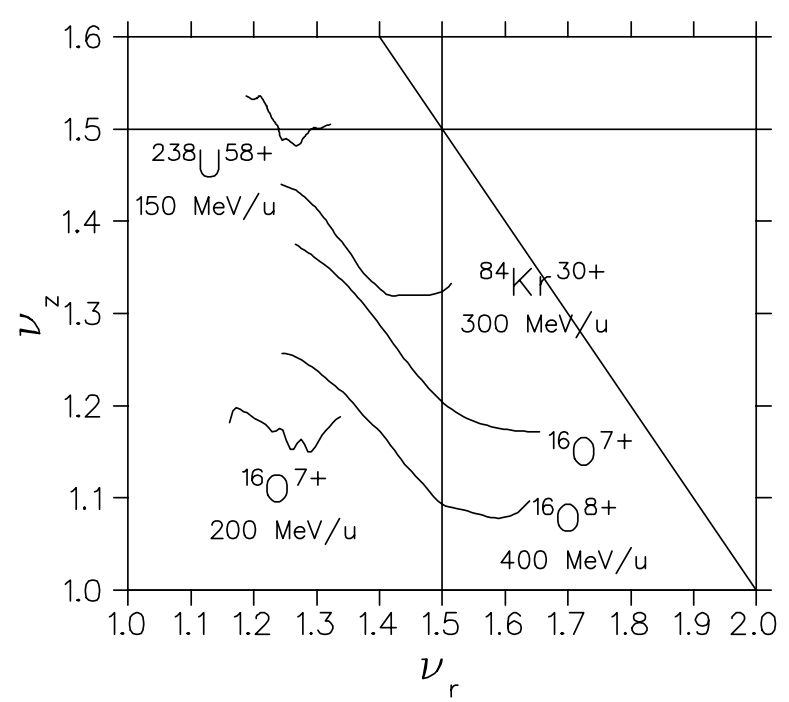

FIG. 1. Tune diagram of several representative ions. Tune plots are smoothed to reduce the ripples contained in the computed fields. 
by ripples in the fitted fields. The integer and half-integer resonances concerned are $\nu_{r}=3 / 2$ for the light nuclei in the final energy of 300-400 MeV/u, and $\nu_{z}=1 / 1$ and $3 / 2$. We could control the vertical tunes by modifying the yoke [6], but the radial resonance must be traversed. The field gradient of a third harmonic Fourier component provokes the $\nu_{r}=3 / 2$ resonance in lowest order. This undesirable harmonic field should be minimized. Independent excitations of the main and some trim coils on each sector can be utilized for compensation of the harmonic field originating from nonuniformity among sector magnets and from the fringe fields of the injection and extraction elements. However, the third harmonic field induced by azimuthal displacements of the magnets cannot be corrected by coil excitations due to a phase mismatching, and thus an elaborate alignment is demanded. Tolerance estimated from optics studies is $0.1 \mathrm{G} / \mathrm{cm}$, which should be practically maintainable.

High-current primary beams are required because only a small fraction of projectile fragments are accepted for use. In particular, when the SRC operates as an injector to the accumulator-cooler ring as currently conceived [1], peak currents in the range of $100 \mathrm{p} \mu \mathrm{A}$ are needed on the pulsed mode. The longitudinal space charge forces will then limit the maximum beam intensity as in other existing high-current cyclotrons [7]. Evaluations have been made on intensity limits assuming simplified beam charge distributions when the beam phase spread is around $20^{\circ}$. In addition, preparations of the beams for injection matching are briefly mentioned. The drifting, thus mismatching, beams should be removed prior to injection to the SRC to reduce beam losses into the extraction elements.

\section{ENHANCED EXTRACTION BY ORBITAL PRECESSION}

A few percent of beam loss into extraction elements may not be tolerable on a localized spot because of wall heating and radiation problems. A high beam power in the SRC calls for single-turn extraction. The beam power amounts to $6.4 \mathrm{~kW}$ for the $400 \mathrm{MeV} / \mathrm{u}{ }^{16} \mathrm{O}$ beam at a current of $1 \mathrm{p} \mu \mathrm{A}$. A cw mode of this beam is requested for the use of nuclear science [8].

A prerequisite to the single-turn extraction is that the orbiting beam acquires a last-turn separation larger than the beamwidth. The turn separation $(\Delta r)$ of a centered beam can be written as

$$
\frac{\Delta r}{d n}=r \frac{\gamma}{\gamma+1} \frac{\Delta E}{E} \frac{1}{\nu_{r}^{2}},
$$

where $r$ is the extraction radius, $\gamma$ is the relativistic factor, $E$ is the kinetic energy of beam, and $\Delta E$ is the energy gain per turn. Once the field strength and geometry of the SRC are outlined, the energy gain determined by the number of arranged cavities and by the peak gap voltage is a prime parameter on the turn separation. The cavity number in the SRC could be three or four interspersed among the six sector magnets. A higher energy gain obtainable with four cavities greatly helps in extraction efficiency and thus in extracted beam current, but the occupation of four valleys imposes some difficulties in laying out the injection and extraction elements. The four cavity scheme is favored for the SRC. However, present orbit tracking was performed assuming the energy gain by three cavities in view of a conservative evaluation. Extension to the case of higher energy gain can be done using the results of this section as illustrated in Sec. V. The main cavity under design is of a single-gap type with a pair of rotatable tuning panels [9]. The maximum design voltage is $600 \mathrm{kV}$ at $38 \mathrm{MHz}$ near the extraction region. The use of a third harmonic flat-topping cavity makes the actual energy gain lower by approximately $12 \%$.

In the SRC the energy gain alone is not sufficient for separating the last turn. In the case of the $400 \mathrm{MeV} / \mathrm{u}$ ${ }^{16} \mathrm{O}^{7+}$ beam, the last-turn separation calculated by Eq. (1) is $2.5 \mathrm{~mm}$ with $\Delta E$ of $1.6 \mathrm{MV} / \mathrm{turn}$. This is the case of the smallest last-turn separation. It becomes $4 \mathrm{~mm}$ with $1.2 \mathrm{MV} /$ turn for the $150 \mathrm{MeV} / \mathrm{u}^{238} \mathrm{U}^{58+}$ beam. The different voltages used reflect different shunt impedances at differing frequencies. On the other hand, the radial width of a matched beam at the injection energy of $126 \mathrm{MeV} / \mathrm{u}$ is about $5 \mathrm{~mm}$ when the emittance is $3.5 \pi \mathrm{mm} \mathrm{mrad}$. This emittance was inferred from that of a $135 \mathrm{MeV} / \mathrm{u}$ beam extracted from the existent RIKEN ring cyclotron (RRC).

As demonstrated with the RRC and other ring cyclotrons [10], one effective method to increase the last-turn separation is to induce orbital precession at the injection. Since the radial tune at the injection is sufficiently larger than unity, a first harmonic field does not cause instabilities in the radial motion, but primarily shifts the centers of beam orbits. When the beam is injected on the equilibrium orbit (EO) determined by the sixfold symmetric main field, a first harmonic field imposed induces precession with a coherent oscillation amplitude proportional to the harmonic field amplitude. In the case of $\mathrm{O}^{7+}$, the first harmonic of $10 \mathrm{G}$ induces precession with an amplitude of $5 \mathrm{~mm}$. This is the case for the maximum last-turn separation.

To investigate the radial motions of eigenellipses when the beam is centered and off-centered, orbit trackings were performed with the SPRGAP orbit code [11]. The code uses the median plane field stored and the off-median plane field computed to first order in $z$. The effect of the flat-topping cavity which breaks the azimuthal symmetry was incorporated into the code. The code can handle an unsymmetric arrangement of cavities, but in the SRC the unsymmetry does not cause a marked orbit distortion because of the high beam energies compared with the energy gain.

Figure 2 shows the phase-space motion of a centered $\mathrm{O}^{7+}$ beam. Radial momentum in the $y$ axis in the figure is given in cyclotron units, and has a dimension of length 

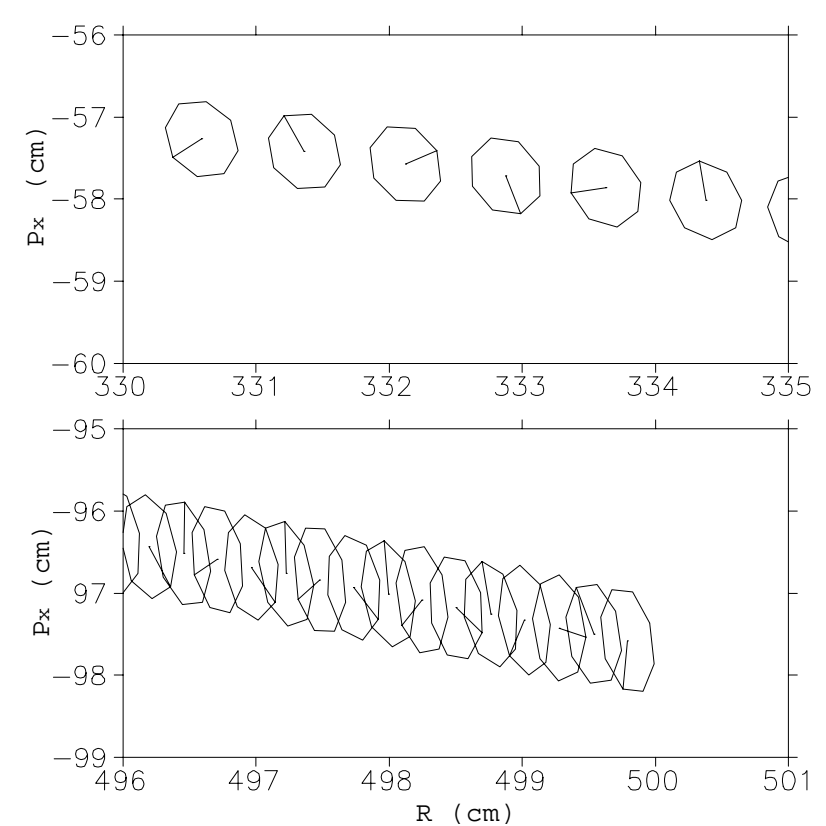

FIG. 2. Upper frame: Phase-space motions of the $400 \mathrm{MeV} / \mathrm{u}$ $\mathrm{O}^{7+}$ beam in the injection region. The plots are made at the azimuth of the electrostatic deflector. Radial momentum $P_{x}$ is given in units of length; values of the ion momenta are divided by $\omega_{0} m_{0}$, where $m_{0}$ is the ion rest mass. The lines drawn inside the phase ellipses are indicators of the radial focusing tunes. Lower frame: Phase space motions in the extraction region.

as the value of the ion momentum is divided by $\omega_{0} m_{0}$, where $m_{0}$ is the ion rest mass. The tracking starts at the middle of the electrostatic inflector channel (EIC), and ends at the entrance of the electrostatic deflector. The plots are made at the azimuth of the deflector entrance. To suppress precession the initial phase ellipse is put on the eigenellipse of an accelerated EO. Turn overlaps at the high-energy end are shown in the figure.

Figure 3 shows the phase-space motion when the orbit is off-centered by $5 \mathrm{~mm}$ at the injection. A correct phase of precession can be attained by adjusting the cavity voltage since the precession phase is chiefly a function of the number of turns $(n)$ as given below:

$$
\phi(n)=\phi_{0}+2 \pi \int_{0}^{n}\left(\nu_{r}-1\right) d n,
$$

where $\phi_{0}$ is the initial phase which varies depending on the way of off-centering, i.e., by the spatial or momentum displacements.

Practical cavities exhibit a radial voltage distribution, so that flat-topping of the rf voltage is not fulfilled in every turn but in average during the acceleration. The cavity voltage profiles were included in orbit simulations. The voltage of the flat-topping cavity was adjusted to minimize the energy spread at the extraction. The voltage distributions of individual cavity were calculated using MAFIA [9] as shown in Fig. 4.

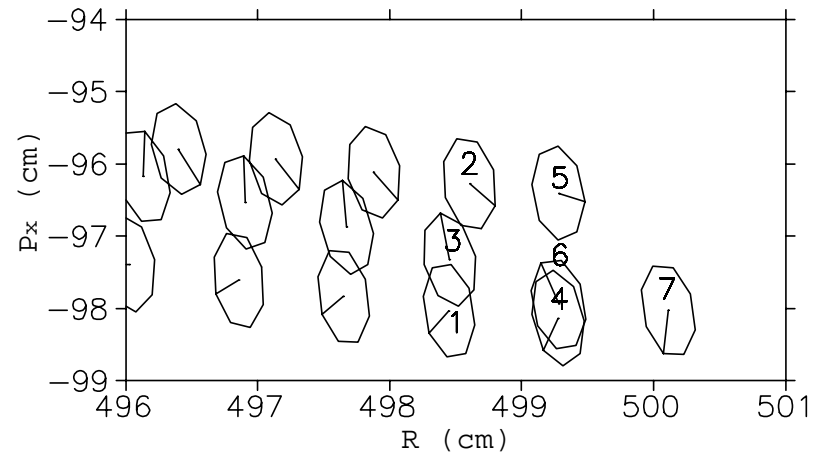

FIG. 3. Phase-space motions when the beam is injected offcentered by $5 \mathrm{~mm}$. Numbering is given to the last seven turns. The precession phase was controlled for maximum separation of the last turn.

Orbit tracking was performed next in six-dimensional phase spaces. The $\mathrm{Z}^{4}$ orbit code [12] was employed because it uses exact equations of motion involving magnetic fields to fourth order in $z$. The code thus evaluates the coupling effect from the vertical to radial phase spaces, which is neglected in SPRGAP. The effect was found, however, to be negligible for the actual vertical acceptance, while the vertical motion itself manifests a small difference from the SPRGAP output in the focusing frequency. Higher-order magnetic fields for the $\mathrm{Z}^{4}$ code were generated using a compact finite difference method [13].

Figure 5 shows the phase spaces of the last five turns which are traced with 200 particles of $\mathrm{O}^{7+}$. The radial and vertical emittances are $3.5 \pi \mathrm{mm} \mathrm{mrad}$, the energy spread $\left(\Delta E_{k} / E\right)$ inferred from the RRC beam is $\pm 0.05 \%$, and the initial phase spread is $20^{\circ}$. Precession is induced at the injection. The result is essentially the same with that attained by the eigenellipse tracing as nonlinear effects are negligible on the given phase spaces.

The design particles for the tracking were randomly populated in six-dimensional phase ellipses. Since the

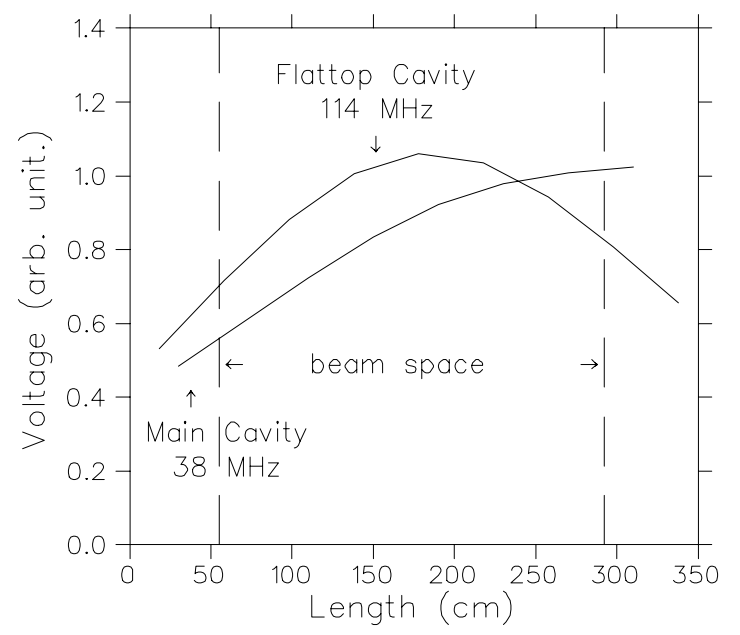

FIG. 4. rf voltage distributions of the main and flat-topping cavities along the length at the highest operation frequencies. 

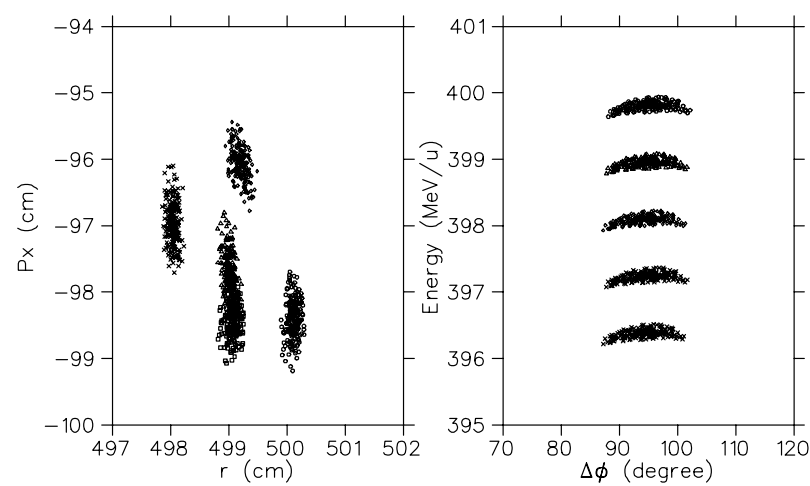

FIG. 5. Phase-space motions of the last five turns of $\mathrm{O}^{7+}$ projected in the transverse and longitudinal phase spaces. The phase spread at the injection was $20^{\circ}$.

beam is radially guided to the SRC as shown in Fig. 6, the actual horizontal and longitudinal phase spaces are correlated. However, no coupling is assumed in the tracking under the condition that the injection elements can be controlled to minimize momentum dispersion at the location of EIC, while the beam phase spaces are matched with any desired phase acceptance. Beam preparations against mismatches are discussed in Sec. VI.

Figure 5 also shows that the phase spread $(\Delta \phi)$ is compressed from $20^{\circ}$ to $15^{\circ}$ at the extraction due to the radially increasing $\mathrm{rf}$ voltage. The relation between $\sin (\Delta \phi)$ and $E$ is given as an approximate invariant by [14]

$$
\Delta E \sin (\Delta \phi)=\text { const. }
$$

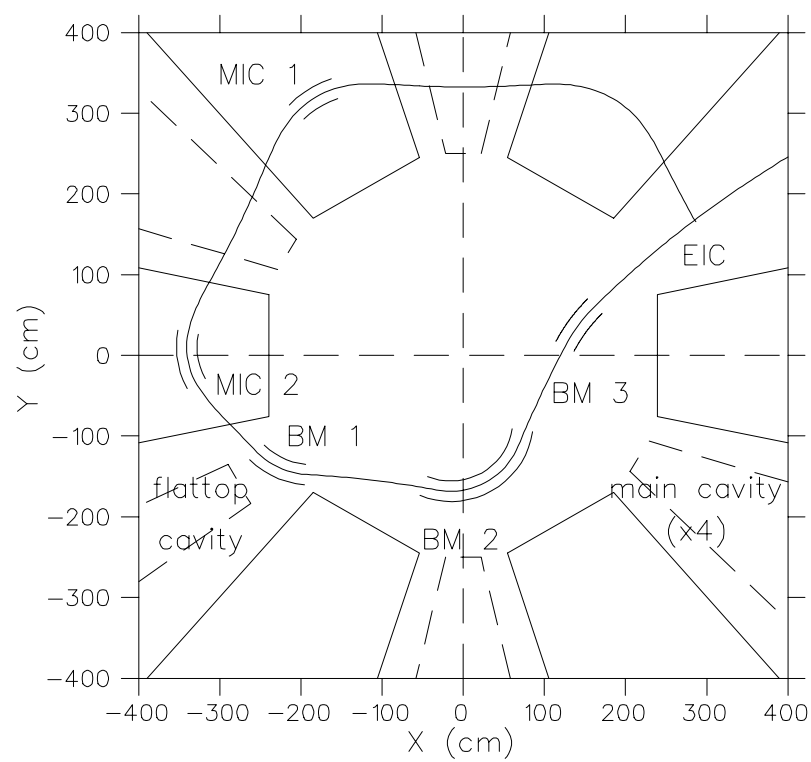

FIG. 6. Injection line into the SRC with the trajectory of the 126.7 $\mathrm{MeV} / \mathrm{u} \mathrm{O}^{8+}$ beam, where $\mathrm{BM}$ stands for the bending magnet, MIC the magnetic inflector channel, and EIC the electrostatic inflector channel. The matching point assumed is the middle of the EIC.
According to the Liouville theorem the energy spread increases with compression of the phase spread. Another energy spread is entailed due to the imperfection of third harmonic flat-topping. The total induced $\Delta E_{k} / E$ for the beam with the initial phase spread of $20^{\circ}$ is less than $\pm 1 \times 10^{-4}$ at the extraction.

Figure 7 shows the radius gain at the extraction versus the energy gain with and without a precession. The precession amplitude can be larger for a higher energy gain. The minimum radius gain increases from 8 to $11 \mathrm{~mm}$ by the use of four cavities.

\section{RADIAL RESONANCE}

When the SRC accelerates the light nuclei above $300 \mathrm{MeV} / \mathrm{u}$, the $\nu_{r}=3 / 2$ resonance is traversed. Gradient of the third harmonic field $B_{3}$ provokes a radial resonance in lowest order. The equation of motion including the perturbing force is given by

$$
\frac{d^{2} x}{d \theta^{2}}+\nu_{r}^{2} x=b x \cos (3 \theta+\phi)
$$

where $b=\frac{r_{0}}{B_{0}} \frac{d B_{3}}{d r}$. As was shown in Ref. [15], the imaginary part of $\nu_{r}$ at the resonance, which accounts for the betatron amplitude growth in the stop band, is derived to be $b / 6$. If the amplitude growth permitted is assumed to be $10 \%$, the tolerable harmonic gradient is calculated as $0.6 \mathrm{G} / \mathrm{cm}$ for the $\mathrm{O}^{7+}$ beam case. This method of estimation is perhaps more applicable when the adiabatic condition on $\nu_{r}$ is well satisfied, such as in a synchrotron. For the cyclotrons in which the resonance is rapidly traversed, a numerical analysis should be more realistic.

A relationship between the bump strength and stopband width on the resonance was studied using the static phase-space trajectories for the flat and gradient bumps. Representative results are displayed for the gradient bump only, while an equivalent bump strength is given to the flat bump. There are three parameters in the case of the

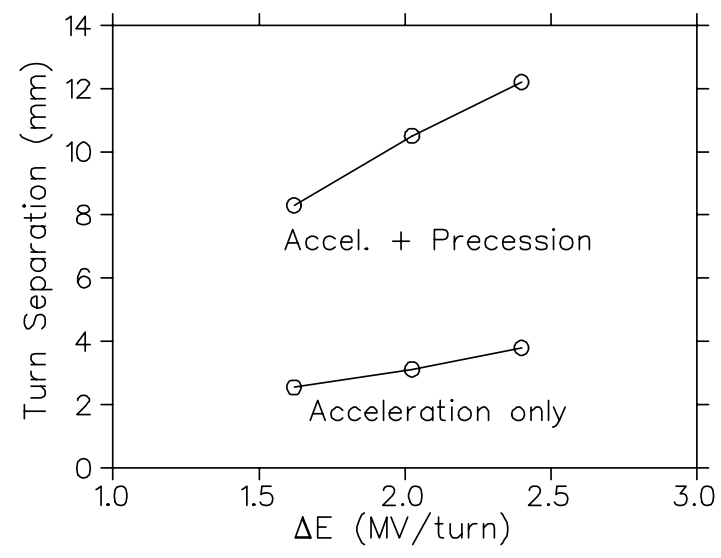

FIG. 7. The turn separation at the extraction versus the cavity voltage when the injected beam is centered (acceleration only) and off-centered (acceleration + precession). 
gradient bump: starting radius, phase angle, and strength. But only two parameters of these are real for the flat bump since the starting radius is not relevant.

Figure 8 shows the phase-space trajectories below and above the resonance when the field gradient of the bump was $0.4 \mathrm{G} / \mathrm{cm}$. The gradient bump crosses the zero field at $490 \mathrm{~cm}$ where the resonance lies, which is arranged to minimize the contribution from the flat component. The plots are made at 306 and $309 \mathrm{MeV} / \mathrm{u}$, where the $\nu_{r}$ 's are 1.496 and 1.503, respectively. A particle makes about 1.5 betatron oscillations per revolution, so that the flow of trajectories on the phase space is antisymmetric with respect to the tilted center line. One important point shown in the figure is a mismatch of eigenellipses across the resonance. The phase of the bump field is selected to match with that of the main flutter field, by which the bump effect becomes largest [16]. When the strength of the gradient bump is compared with that of the flat bump under a same stop-band width, $0.4 \mathrm{G} / \mathrm{cm}$ is about equivalent to $35 \mathrm{G}$.

To clarify the effects of the resonance crossing in phase spaces, an eigenellipse of the $\mathrm{O}^{7+}$ beam was traced in the field with a prescribed third harmonic gradient. Figure 9 shows the case when the imposed field-gradient bump was $0.7 \mathrm{G} / \mathrm{cm}$ with and without an off-centering injection (a stronger bump than the previous one is used simply to magnify the effect of the bump). The phase space matched at the injection oscillates beyond the resonance as being implied by the static phase plots. The offcentering injection still enlarges the last-turn separation, but is less effective. The separation between the last two turns disappears even with precession when the gradient of $B_{3}$ is about $1.0 \mathrm{G} / \mathrm{cm}$. Further, transmission efficiency through the extraction channels decreases as the radial spread of the beam becomes larger. Note that the entrance width of the deflector is designed to be around $10 \mathrm{~mm}$.

It is viewed that the gradient field should be kept sufficiently below $0.4 \mathrm{G} / \mathrm{cm}$ for the beams accelerating
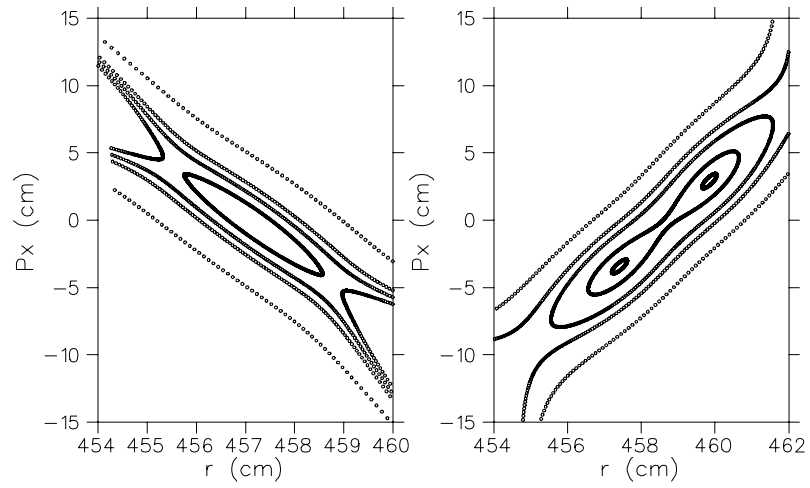

FIG. 8. Static phase-space trajectories below and above the resonance shown in the left- and right-hand sides, respectively, when the perturbing third-harmonic gradient is $0.4 \mathrm{G} / \mathrm{cm}$. Beam energies of $\mathrm{O}^{7+}$ are 306 and $308 \mathrm{MeV} / \mathrm{u}$, where the tunes are 1.496 and 1.503 , respectively. to $400 \mathrm{MeV} / \mathrm{u}$. The tolerance conservatively estimated is $0.1 \mathrm{G} / \mathrm{cm}$. Harmonic excitations of the main coils and of outer trim coils placed with an interval of $10 \mathrm{~cm}$ on each sector magnet will be utilized to remove the major portion of a third-harmonic gradient [17]. The remnant gradient components due to the limited field form factors and phase angles of the coil excitations should be negligible. On the other hand, tolerance is higher for the beams traversing the resonance at a larger radius; e.g., for the $300 \mathrm{MeV} / \mathrm{u}$ ${ }^{84} \mathrm{Kr}^{30+}$ beam. The tolerable gradient bump is estimated to be roughly 1.5 times stronger for this beam.

\section{VERTICAL RESONANCES}

If the mirror symmetry in a cyclotron is violated, horizontal components of the field would appear on the midplane. Fourier harmonics of $B_{r}$ can then induce a beam blowup at relevant vertical resonances. To simulate the vertical alignment errors of cold mass in a sector magnet, which could be a major source of the mirror symmetry breaking, the harmonic components of $B_{r}$ with no vertical dependence were included in the orbit tracking with the $\mathrm{Z}^{4}$ code. Since the pole gap width of $40 \mathrm{~cm}$ is much larger than the beam height, a vertically uniform $B_{r}$ should be a reasonable approximation.

As shown on the tune diagram in Fig. 1, the $\nu_{z}=3 / 2$ is traversed while the $\nu_{z}=1$ is kept away. This tune arrangement is due to the fact that the integer imperfection resonance is more destructive than the half-integer one. However, prior to the field mapping of the arranged six sector magnets, it is rather difficult to predict the exact values of the minimum $\nu_{z}$. In principle, the field flutter could be adjusted to control the $\nu_{z}$, for instance,

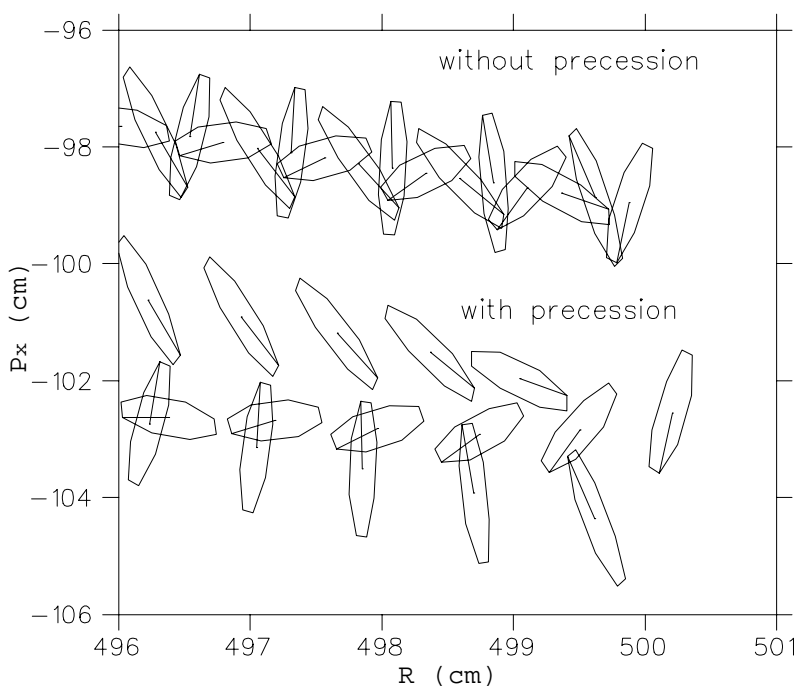

FIG. 9. Phase-space motions with and without a precession near the extraction region. The third harmonic gradient of $0.7 \mathrm{G} / \mathrm{cm}$ is imposed. Because of the mismatch of eigenellipse with a new one above the resonance, the beam phase space oscillates. 
by shimming the yoke after the field mapping. But in practice, the $\nu_{z}$ could be kept sufficiently larger than unity in the initial design.

The $\nu_{z}$ of the ions with $q / A=0.5$ is 1.07 under the design field at the energy around $390 \mathrm{MeV} / \mathrm{u}$, and is 1.08 at $400 \mathrm{MeV} / \mathrm{u}$. The first harmonic $B_{r}$ near the $\nu_{z}=1$, its presence being everywhere in the cyclotron, induces a vertically coherent oscillation whose amplitude $\left(z_{e o}\right)$ is given if the smooth approximation is used by

$$
z_{e o}(\omega t)=r \frac{\left\langle B_{r}\right\rangle}{\left\langle B_{z}\right\rangle} \frac{1}{\nu_{z}^{2}-1} \cos (\omega t+\phi),
$$

where the bracket indicates an azimuthal average. The actual oscillation amplitude at a given energy depends on the radial profile of the $B_{r}$ chiefly due to the orbit scalloping. The vertical envelopes are calculated as shown in Fig. 10 along the azimuth of the valley center for three cases of different profiles and magnitudes, illustrated in the inset graph. The vertical beam height in use is $\pm 5 \mathrm{~mm}$, while it is $\pm 3 \mathrm{~mm}$ for the beam with an emittance of $3.5 \pi \mathrm{mm}$ mrad. When the $B_{r}$ is flat and equal to $1 \mathrm{G}$ as indicated by the solid line, the orbit center is vertically shifted by $5 \mathrm{~mm}$ for an injection matching, for which the $z_{e o}$ near $\nu_{z}=1$ agrees with that calculated with Eq. (5).

Considering that the half height of the beam chamber is $3 \mathrm{~cm}$, the first harmonic $B_{r}$ should be kept much smaller than $10 \mathrm{G}$ if the minimum $\nu_{z}$ is 1.07 . For a lower minimum $\nu_{z}$, the tolerable field level can be obtained by using the scaling of Eq. (5). On the other hand, note that the first harmonic $B_{r}$ generated with a $1 \mathrm{~mm}$ vertical shift of the cold mass in a single sector magnet is about $0.6 \mathrm{G}$, and the alignment tolerance is set to be $\pm 1 \mathrm{~mm}$. As a result, the specified alignment tolerance could be applicable up to the minimum $\nu_{z}$ of 1.02. The vertical motion affected by an orbital precession was investigated next, but no notable change was observed within the offcenteredness required for single-turn extraction.

The ions with $q / A$ near 0.25 traverse the $\nu_{z}=1.5$ resonance. The $150 \mathrm{MeV} / \mathrm{u} \mathrm{U}^{58+}$ beam was used for

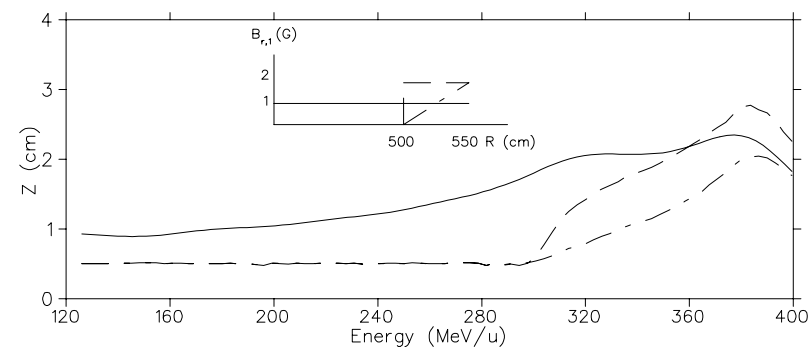

FIG. 10. Vertical envelopes of the $\mathrm{O}^{7+}$ beam accelerating from 128 to $400 \mathrm{MeV} / \mathrm{u}$ with the first harmonic $B_{r}$ of three different profiles: (1) the solid line is for a uniform $1 \mathrm{G} B_{r}$ field, (2) the dashed line is for a $2 \mathrm{G}$ step $B_{r}$ field starting at a radius of $500 \mathrm{~cm}$, and (3) the dot-dashed line is for a slanted $B_{r}$ field also started at $500 \mathrm{~cm}$. The plots are made at the center of the valley. envelope calculations since its $\nu_{z}$ hovers around 1.5 . Optics calculations were carried out for the flat field and gradient field errors of $10 \mathrm{G}$ and $0.5 \mathrm{G} / \mathrm{cm}$, respectively. It turned out that the amplitudes of coherent oscillation are much smaller than the height of the beam chamber. Traversal of the $\nu_{z}=1.5$ appears safe insofar as the alignment tolerance is maintained.

\section{SPACE CHARGE EFFECTS}

In the RI beam factory under construction at RIKEN, the variable frequency RFQ and Wideröe accelerators are the injectors for the cyclotron chain composed of three cyclotrons: RRC, IRC, and SRC [1]. The space charge limiting beam current in the linear accelerators is usually higher than in the isochronous cyclotrons owing to the provision of both transverse and longitudinal focusing forces. In the cyclotrons the longitudinal focusing is almost absent, so as to limit the maximum beam intensity.

While the IRC and SRC, which are the boosters of the RRC, are to be equipped with flat-topping cavities, the RRC is not. Hence the space charge forces at high currents cannot be mitigated by a broad phase spread of beam. A recent measurement showed that the phase spread is about $5 \mathrm{rf}$ degrees when the energy spread is $1 \times 10^{-3}$ for the $90 \mathrm{MeV} / \mathrm{u}{ }^{46} \mathrm{Ti}$ beam at a low current [18]. Since the last-turn separation at the RRC is much larger than at the SRC (about $1 \mathrm{~cm}$ at the RRC compared to $2.5 \mathrm{~mm}$ at the $\mathrm{SRC}$ for the centered $\mathrm{O}^{7+}$ beam), it seems that the beams with a larger energy spread can be extracted at the cost of beam quality. The beam current aimed during Phase I of the project is $1 \mathrm{p} \mu \mathrm{A}$ in $\mathrm{cw}$ mode for light nuclei. The space charge forces in the RRC may not be a limiting factor (Ar beam of $1 \mathrm{p} \mu \mathrm{A}$, for instance, has been extracted from the RRC [19]), but for Phase II where much higher current in the range of $100 \mathrm{p} \mu \mathrm{A}$ is requested the space charge issues should be readdressed along with relevant measurements.

The limiting current here is defined as the current when the last-turn separation disappears due to beam energy spreads induced by longitudinal space charge forces. To compute the space charge forces, the charge distribution of the beam in the cyclotron should be known, Actual charge distributions are complicated especially when the beam orbit is in precession for single-turn extraction as seen in Figs. 3 and 5. For first-order evaluations without actual integration of all orbits, separated turns were assumed in calculating the charge densities in a beam, so that variations in the local charge densities due to noncentered orbital motion are not considered.

The azimuthal electric field which induces energy spreads has been calculated using two different methods. The first method considers the model of a uniformly charged disk inside a sector shape beamlet. Then the electric field at the azimuthal edges of a bunch can be 
given by $[7,20]$

$$
E_{\theta}=\frac{\rho a}{2 \epsilon_{0}} g
$$

where $a$ is a half-width of the vertical beam height, $g$ is the form factor, and $\rho$ is the charge density. For the $\mathrm{O}^{7+}$ beam, $a$ is roughly $0.25 \mathrm{~cm}$, and $g$ is 1.4. The $E_{\theta}$ averaged in between the injection and extraction ports is then calculable to obtain $9 \mathrm{~V} / \mathrm{m}$ at a current of $1 \mathrm{p} \mu \mathrm{A}$. The total energy spread accumulated throughout 450 turns is about $\pm 54 \mathrm{kV} / \mathrm{u}$. Since the lastturn separation disappears if $\Delta E_{k} / E$ becomes larger than about $2 \times 10^{-3}$ in the case of three cavities, the limiting current can be estimated to be $10 \mathrm{p} \mu \mathrm{A}$ providing that the energy spread inherent and induced by other mechanisms is $4 \times 10^{-4}$ during acceleration.

The second more elaborate method was devised by Gordon [21], which can compute the potential of a charged beam as a function of the azimuth for a given charge distribution. This model includes the effect of image charges due to the beam chamber. The charge distribution at the time of injection is not known to us when the phase spread is $20^{\circ}$. Therefore, the azimuthal electric fields were calculated assuming two simplified shapes of distribution: triangular and rectangular shapes. The electric field as a function of $\phi$ is given as

$$
E_{\theta}(\phi)=2 F_{0} G(\phi) \gamma^{3}
$$

where $F_{0}$ is the quantity having an electric field unit and $G(\phi)$ is a dimensionless field function specified by the geometric parameters. For the $\mathrm{O}^{7+}$ beam, the $F_{0}$ averaged during acceleration is calculated to be about $2 \mathrm{~V} / \mathrm{m}$. $G(\phi)$ is plotted in Fig. 11 for the two kinds of charge distributions having the phase spreads of $20^{\circ}$ and $15^{\circ}$ at the injection and extraction, respectively. The vertical beam height was assumed to be $6 \mathrm{~mm}$ with a rectangular charge distribution. Conducting planes are at $z= \pm 3 \mathrm{~cm}$. If the actual maximum electric field is an average of the two kinds of distributions, the maximum electric field at $1 \mathrm{p} \mu \mathrm{A}$ is about $13 \mathrm{~V} / \mathrm{m}$, which gives the limiting current $7 \mathrm{p} \mu \mathrm{A}$.

According to the scaling to a different energy gain under the assumption of separate turns, the intensity limit increases by $(4 / 3)^{2}$ times with the adoption of four cavities, instead of three, due to the reduction of the number of turns and due to the increase of the turn separation. The third power dependence of the energy gain predicted by the disk model [7,22] is due to the consideration of changes in the beam charge density.

Energy spreads of other ion beams could be estimated from the dependence of $q^{2} / A$ for the same energy gain. Hence, heavy nuclei in higher charge states are more vulnerable to the space-charge forces. For instance, the limiting current of the $\mathrm{U}^{58+}$ could be smaller more than a factor of 10 than that of the $\mathrm{O}^{7+}$ if their energy gains are given as described in Sec. II. The current aimed for heavy nuclei is in the range of $100 \mathrm{pnA}$.

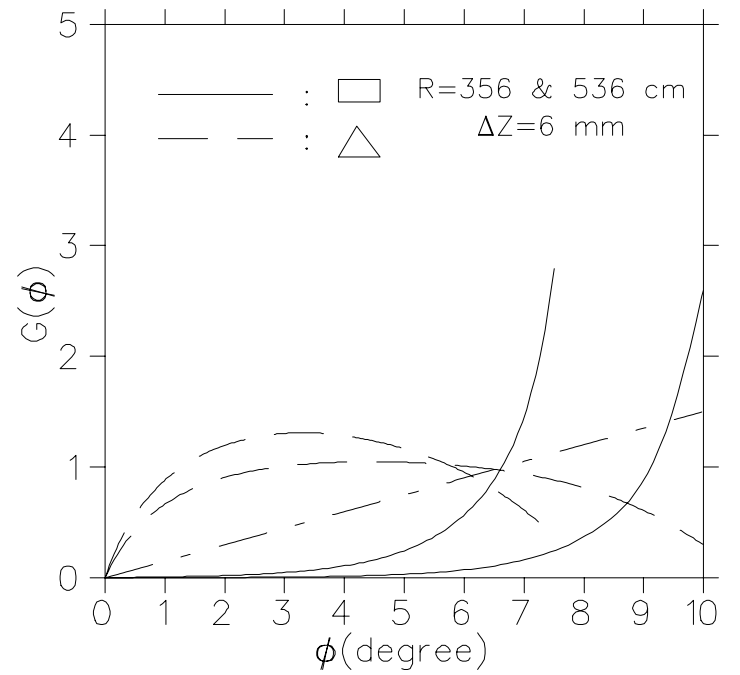

FIG. 11. Plots of the electric field form $G(\phi)$ at the injection and extraction for the rectangular and triangular charge distributions in the azimuthal direction. The vertical distribution was assumed to have a rectangular shape. The vertical beam size in use is $6 \mathrm{~mm}$ with conduction planes at $z= \pm 3 \mathrm{~cm}$. $G(\phi)$ is assumed to be symmetric with respect to the central phase. The dot-dashed line indicates the correction of $E_{\theta}$ by rf phase tuning for the rectangular distribution.

A measurement at the RRC showed that the charge distribution versus of phase was quite symmetric across the central phase [18]. If the symmetry continues up to high currents with the phase spread around $20^{\circ}$, the linear part of the longitudinal space charge forces can be corrected by tuning the relative phase $(\varphi)$ between flattopping and main cavities. The dot-dashed line in Fig. 11 shows a linear correction along the azimuth of the beam bunch for the case of rectangular charge distribution. If the correction is valid, the limiting current could be roughly doubled as the maximum electric field reduces by half. A similar gain may be expected depending on the charge distributions of actual beam. The energy gain per turn including the flat-topping voltage $\left(V_{3}\right)$ is given by

$$
\Delta E=V \sin (\phi)+V_{3} \sin (3 \phi+\varphi) .
$$

For the SRC operating with three cavities, the magnitude of $\varphi$ needed for adjustment is $0.035^{\circ} / \mathrm{kV} / \mathrm{u}$, which indicates that the phase stability among the cavities is seriously important.

\section{INJECTION MATCHING}

To reduce the beam losses at the high-energy end of SRC, an unmatched portion of the beam in sixdimensional phase space should be removed prior to the injection. The injection line is to be designed to meet the matching requirements for different ions, but in practice a mismatch can occur for instance by machine instabilities in the accelerator chain. If a beam which was disturbed in the transverse phase space reaches the SRC, a part 
of the beam could be lost in the injection line. The injection elements will be protected against stray beams such as water-cooled shields. A more active scheme is to install slit systems to define transverse phase spaces for screening the halo and drifting beams. The injection line is to be designed to accommodate the slits. The longitudinal phase stability, on the other hand, can be monitored with beam phase measurement [23]. The flattopping cavities in the IRC and SRC and the bunchers to be installed in the beam transfer line could help control the central phase and compensating changes in beam phase spreads.

Instabilities in SRC and its components will affect the beam in acceleration. Beam losses may abruptly occur inside the cyclotron. Overall system protection on the SRC will be ensured by measuring the current difference between injection and extraction, and by radiation monitoring.

\section{CONCLUSION}

The conditions to achieve a single-turn extraction in the SRC were evaluated. In addition, the intensity limit imposed by longitudinal space charge forces was investigated, and preparations for the injection matching of beams were discussed. The extraction efficiency and limiting current can be greatly enhanced with the use of four cavities instead of three in the six-sector SRC. The adoption of four cavities is surely favored, but availability of space is a problem. The arrangement of cyclotron components then becomes critical not to cause interference among them. Optics simulation tells that the turn separation by three cavities is actually sufficient to achieve extraction efficiency of over $99 \%$ for a Gaussian beam with an emittance of $3.5 \pi \mathrm{mm}$ mrad at a current of $1 \mathrm{p} \mu \mathrm{A}$. However, the use of four cavities will approximately double the intensity limit. The limiting current of the $\mathrm{O}^{7+}$ beam with four cavities was estimated to be in the range of $10-20 \mathrm{p} \mu \mathrm{A}$. The six-dimensional phase spaces of different ion species should be matched with cyclotron acceptance at the EIC. A complete design of the injection line involves the knowledge of incoming beam properties determined by the whole accelerator chain, which is not yet available. Moreover, slit systems for the selection of transverse emittance should be installed in the transport line in order to prevent beam-induced damages on the extraction components.

[1] Y. Yano, A. Goto, and T. Katayama, in Proceedings of the 1997 Particle Accelerator Conference (IEEE, Piscataway, NJ, 1998), p. 930.

[2] T. Kawaguchi et al., in Proceedings of the 15th International Conference on Cyclotrons and Their Applications (to be published).

[3] W. M. Schulte, H. L. Hagedoorn, and C. J. A. Corsten, IEEE Trans. Nucl. Sci. NS-26, 2392 (1979).

[4] J. Kim, et al., in Proceedings of the 1997 Particle Accelerator Conference (Ref. [1]), p. 3422.

[5] TOSCA is a product of Vector Fields, Oxford, England.

[6] T. Mitsumoto et al., in Proceedings of the 15th International Conference on Cyclotrons and Their Applications (to be published).

[7] W. Joho, in Proceedings of the 9th International Conference on Cyclotrons and Their Applications (Editions de Physique, Les Ulis, France, 1982), p. 337.

[8] I. Tanihata, Nucl. Phys. A616, 56 (1997).

[9] N. Sakamoto et al., RIKEN Accel. Prog. Rep. 31, 222 (1998).

[10] W. Joho and D. Adam, IEEE Trans. Nucl. Sci. NS-26, 2358 (1979).

[11] M. M. Gordon, Nucl. Instrum. Methods 169, 327 (1980).

[12] M. M. Gordon and V. Taivassalo, Nucl. Instrum. Methods Phys. Res., Sect. A 247, 423 (1986).

[13] D. Jeon, J. Comput. Phys. 132, 167 (1997).

[14] W. Joho, Part. Accel. 6, 41 (1974).

[15] M. M. Gordon, Ann. Phys. (N.Y.) 50, 571 (1968).

[16] D. I. Hopp and J. R. Richardson, Nucl. Instrum. Methods 44, 277 (1966).

[17] J. Kim et al., in Proceedings of the 15th International Conference on Cyclotrons and Their Applications (to be published).

[18] H. Kumagai, Internal note, Linear Accelerator Laboratory, RIKEN (1998).

[19] O. Kamigaito et al., RIKEN Accel. Prog. Rep. 31, 192 (1998).

[20] W. Joho, Int. Accelerator School, Dubna, USSR, 1988.

[21] M.M. Gordon, in Proceedings of the 5th Cyclotron Conference (Butterworths, London, 1969), p. 305.

[22] Th. Stammbach et al., Nucl. Instrum. Methods Phys. Res., Sect. B 113, 1 (1996).

[23] T. Kawama et al., RIKEN Accel. Prog. Rep. 27, 135 (1993). 